\title{
Charge qubit entanglement via conditional single electron transfer in an array of quantum dots
}

\author{
A V Tsukanov \\ Institute of Physics and Technology, Russian Academy of Sciences, Nakhimovsky \\ prospect 34, Moscow 117218, Russia \\ E-mail: tsukanov@ftian.ru
}

\begin{abstract}
We propose a novel scheme to generate entanglement among quantumdot-based charge qubits via sequential electron transfer in an auxiliary quantum dot structure whose transport properties are conditioned by qubit states. The transfer protocol requires the utilization of resonant optical pulses combined with appropriate voltage gate pattern. As an example illustrating the application of this scheme, we examine the nine-qubit Shor code state preparation together with the error syndrome measurement.

PACS numbers: 03.67.Mn, 03.67.Pp, 73.23.-b, 78.67.-n
\end{abstract}

Submitted to: J. Phys. C: Solid State Phys. 


\section{Introduction}

A wide class of problems concerned with the quantum information processing requires an efficient and robust methods of the creation of highly-entangled states from individual qubit states [1], 2]. Therefore, the search and the development of reliable schemes for entanglement production appears to be a rather important quantum computational issue. Recently, several proposals for the entanglement generation in the semiconductor nanostructures based on the quantum dots (QDs) (see, e. g., Refs. [3] - 6]) and its application (see, e. g., Refs. [7] - [13]) have been made. In those works, the fault-tolerant quantum algorithms [7] - [9], the mechanisms of the quantum teleportation [10] - [12], and the cluster state preparation in the measurement-based quantum computations [13] exploit specific entangled charge or spin states of electrons bound in the QDs.

In particular, the logical states of an individual (physical) qubit can be presented by two single-electron orbital states localized in the spatially separated potential minima of a double quantum dot (DQD) [14] - [22]. The quantum operations on that socalled charge qubit can be accomplished via adiabatic variation of the DQD confinement potential [14 - 17] or by the electromagnetically induced resonant transitions between the DQD states [18] - 222. In order to perform some non-trivial two-qubit operation on an arbitrary pair of qubits in a quantum register one should organize the interaction between those qubits for a finite period of time. In general, after interaction is off, the qubits become entangled with each other, i. e., their total wave function cannot be presented as the product of an individual qubit wave functions. The charge qubit entanglement via electrostatic control over the tunnel coupling between two neighboring qubits has been studied in Refs. [4], [6], [11], [14] - [17].

In this paper, we suggest another technique of entanglement production in the twodimensional array of the charge qubits. The coupling between the qubits is now indirect and mediated by an auxiliary structure (AS) fabricated from the quasilinear chains of the QDs and containing a single (probe) electron in the quantized part of its conduction band. The single-electron energy spectrum of that structure is affected by qubit states through the electrostatic interaction between the probe and qubit electrons. In order to demonstrate how to entangle the qubits we analyze in detail the probe electron dynamics in a separate quasilinear QD AS capacitively coupled to the charge qubit. As we shall see, appropriate variation of the confinement potential of such an AS through the application of compensating voltages aligns the energy levels of individual AS QDs

for one of two logical qubit states. It amounts to sharp increase of the tunnel coupling between the AS QDs that, in its turn, allows for efficient probe electron transfer (PET) along the AS through one of the hybridized AS states (the transport state). The transfer protocol is accomplished via the resonant transitions connecting two states each localized in corresponding edge $\mathrm{AS} \mathrm{QD}$ and the transport state delocalized over the AS. The numerical simulation of electron dynamics under tight-binding approximation confirms the possibility of successful PET implementation.

Two points are important here. Firstly, PET from one edge AS QD to another and 
back results in the implementation of the phase operation on attached qubit. Secondly, the PET between the edge AS QDs takes place only if the qubit is in the predetermined logical state. Therefore, the following dynamics of probe electron appears, in its turn, to be predetermined (conditioned) by the qubit state. It enables us to organize conditional quantum operations (in particular, controlled-phase operation) that is the key part of entangled state preparation. Using these results and assuming that PET in a complex planar AS can be divided into the sequence of elementary PETs along its quasilinear components, it becomes possible to construct any desired entangled state of the charge qubits attached to that AS. Particularly, the set of described manipulations underlying controlled-phase operations, combined with single qubit Hadamard rotations, is sufficient for the nine-qubit Shor encoding procedure [23]. Moreover, the conditional PET may be exploited for an error syndrome measurements and for usual qubit state measurement as well.

The paper is organized as follows. In Section 2 we examine in detail the energy spectrum of the auxiliary QD structure attached to single qubit as well as the organization of conditional probe electron dynamics in that structure. This specific operation amounts to a single-qubit phase shift and can be used for implementation of controlled-phase shift of target qubit in two-qubit circuits and, therefore, for entanglement generation. Section 3 describes the algorithm of nine-qubit Shor encoding which exploits two-qubit gates based upon conditional probe electron evolution to entangle the qubits. Auxiliary structure used in the algorithm is arranged from quasilinear QD chains considered in Section 2. We conclude our study by Section 4.

\section{The conditional electron transfer along a quasilinear chain of quantum $\operatorname{dots}$}

In this Section we study a way of how to manipulate the two-electron system composed of charge qubit and auxiliary structure by selective driving of one of its components, namely, of the probe electron localized in the AS. As we shall see, the probe electron evolution in the AS depends on the qubit state, and, in its turn, may change internal qubit phase. Both aspects are important for an algorithm of entanglement production in many-qubit systems.

\subsection{The model and stationary eigenstates}

Consider the single-electron AS composed of $N$ electrostatically defined QDs $A_{k}(k=$ $1-N)$ that are stacked in a quasilinear chain and separated by the finite potential barriers (figure 1). The structure parameters are chosen in such a way that the edge QD $A_{1}\left(A_{N}\right)$ contains at least two bound electron states, namelly, the well-localized ground state $\left|g_{1}\right\rangle\left(\left|g_{N}\right\rangle\right)$ and the excited state $\left|e_{1}\right\rangle\left(\left|e_{N}\right\rangle\right)$ lying closely to the barrier's top, while each internal QD $A_{k}[k=2-(N-1)]$ contains at least one bound electron state $\left|e_{k}\right\rangle$. The QDs $A_{1}$ and $A_{N}$ are supposed to be spatially isolated from each other so that the 
electron tunneling between the states $\left|g_{1}\right\rangle$ and $\left|g_{N}\right\rangle$ is negligibly small. If an electron occupies one of those states, it can stay there for extremely long time. We assume that the energies $\varepsilon\left(e_{1}\right)$ and $\varepsilon\left(e_{N}\right)$ of excited states of the edge QDs and the energies $\varepsilon\left(e_{k}\right)$ of states of the internal QDs are approximately equal to each other. Given the tunnel coupling $\tau_{k}\left(\tau_{k}>0\right)$ between the states $\left|e_{k}\right\rangle$ and $\left|e_{k+1}\right\rangle$ is large as compared with the energy difference $\left|\varepsilon\left(e_{k}\right)-\varepsilon\left(e_{k+1}\right)\right|$ (resonant tunneling condition), those states become hybridized. If this condition holds for any neighboring AS QD states $\left|e_{k}\right\rangle$ and $\left|e_{k+1}\right\rangle$, then the single electron tunneling between QDs results in formation of a $N$-fold excited state subband. Each state in that subband is a superposition of states $\left|e_{k}\right\rangle(k=1-N)$ and is thus delocalized over the AS. Here we imply that the ground states of edge QDs do not hybridize with the excited states as long as $\varepsilon\left(e_{1(N)}\right)-\varepsilon\left(g_{1(N)}\right) \gg \tau_{1(N-1)}$.

If charge qubit is positioned along the structure axis $x$, as shown in figure 1 , the energies of the AS QD levels are shifted relative to their unperturbed values $\varepsilon\left(g_{1}\right)$, $\varepsilon\left(g_{N}\right)$, and $\varepsilon\left(e_{k}\right)$ by $U_{q}\left(g_{1}\right), U_{q}\left(g_{N}\right)$, and $U_{q}\left(e_{k}\right)$, respectively, due to the electronelectron interaction. For definiteness, those shifts are completely associated with bare single-electron levels of the AS QDs. The shifts depend on the logical qubit state $|q\rangle$ $(q=0,1)$ and result in the suppression of the single electron tunneling between the neighboring AS QDs $A_{k}$ and $A_{k+1}$ provided that the electrostatically induced energy mismatch for individual QD states is larger than the corresponding tunneling matrix element, i. e., $\left|U_{q}\left(e_{k}\right)-U_{q}\left(e_{k+1}\right)\right| \geq \tau_{k}$. In order to eliminate the electrostatic energy shifts $U_{q}\left(e_{k}\right)$ of the exited QD levels and to recover the resonant character of the electron tunneling along the AS, one can apply to each AS QD $A_{k}$ the voltages that generate the energy shifts $\delta U_{q}\left(e_{k}\right)=-U_{q}\left(e_{k}\right)$. Obviously, the sets $\left\{\delta U_{0}\left(e_{k}\right)\right\}_{k=1}^{N}$ and $\left\{\delta U_{1}\left(e_{k}\right)\right\}_{k=1}^{N}$ of compensating voltages for two logical charge qubit states $|0\rangle$ and $|1\rangle$ are different. For example, if one uses the voltage set corresponding to the qubit state "one" and $\left|\left[U_{1}\left(e_{k}\right)-U_{0}\left(e_{k}\right)\right]-\left[U_{1}\left(e_{k+1}\right)-U_{0}\left(e_{k+1}\right)\right]\right| \geq \tau_{k}$ for some $k$, the unperturbed AS excited energy subband is reconstructed only if the qubit state is "one". Hereafter, we shall neglect the dependency of tunneling matrix elements $\tau_{k}$ on the qubit state assuming that corresponding variations in the interdot barrier heights have small effect on $\tau_{k}$. In general, one can control the values of $\tau_{k}$ with the voltages on the gates defining the barriers.

We analyze our two-electron system within the tight-binding approximation and restrict ourselves to the case that the qubit electron is always localized in the logical subspace of the DQD. Thus the product states $\left|g_{1}, q\right\rangle=\left|g_{1}\right\rangle|q\rangle,\left|g_{N}, q\right\rangle=\left|g_{N}\right\rangle|q\rangle$, $\left|e_{1}, q\right\rangle=\left|e_{1}\right\rangle|q\rangle, \ldots,\left|e_{N}, q\right\rangle=\left|e_{N}\right\rangle|q\rangle(q=0,1)$ can be used as the basis states. In this case, the Hamiltonian describing the stationary two-electron states reads

$$
H=H_{A S}+H_{q}+H_{A S-q}
$$

where $H_{A S}=\varepsilon\left(g_{1}\right)\left|g_{1}\right\rangle\left\langle g_{1}\left|+\varepsilon\left(g_{N}\right)\right| g_{N}\right\rangle\left\langle g_{N}\left|+\sum_{k=1}^{N} \varepsilon\left(e_{k}\right)\right| e_{k}\right\rangle\left\langle e_{k}\right|-\sum_{k=1}^{N-1}\left[\tau_{k}\left|e_{k}\right\rangle\left\langle e_{k+1}\right|+h . c.\right]$ is the AS Hamiltonian, $H_{q}=\varepsilon_{0}|0\rangle\left\langle 0\left|+\varepsilon_{1}\right| 1\right\rangle\langle 1|$ is the qubit Hamiltonian, and the term

$$
H_{A S-q}=\sum_{q=0,1}\left[U_{q}^{\prime}\left(g_{1}\right)\left|g_{1}, q\right\rangle\left\langle g_{1}, q\left|+U_{q}^{\prime}\left(g_{N}\right)\right| g_{N}, q\right\rangle\left\langle g_{N}, q\left|+\sum_{k=1}^{N} U_{q}^{\prime}\left(e_{k}\right)\right| e_{k}, q\right\rangle\left\langle e_{k}, q\right|\right]
$$


accounts for the effective interaction between the probe and qubit electrons. (In our model, the tunneling between the AS and the qubit as well as the non-diagonal electrostatic terms are completely ignored.) Here $U_{q}^{\prime}\left(e_{k}\right)=U_{q}\left(e_{k}\right)+\delta U_{q^{\prime}}\left(e_{k}\right)$ and we assume that $U_{q}^{\prime}\left(g_{1}\right) \approx U_{q}^{\prime}\left(e_{1}\right), U_{q}^{\prime}\left(g_{N}\right) \approx U_{q}^{\prime}\left(e_{N}\right)$. The prime at $U_{q}^{\prime}\left(e_{k}\right)$ indicates on that the external voltages generate the shifts $\delta U_{q^{\prime}}\left(e_{k}\right)$ compensating the electrostatic shifts provided that $q=q^{\prime}$. The electrostatic coupling energies are taken in the form

$$
U_{q}\left(e_{k}\right)=\frac{U_{0}}{1+(1-q) L / r_{0}+(N-k) r_{c} / r_{0}},
$$

where $U_{0}=1 / r_{0}, r_{0}$ is the distance between the center of the AS QD $A_{N}$ and the nearest minimum of the DQD corresponding to the qubit state "one", $L$ is the distance between the potential minima of the DQD, and $r_{c}$ is the distance between the centers of neighboring AS QDs. For simplicity, the AS QDs are supposed to be equally spaced from each other. In what follows, we shall consider the AS composed of $N=20$ QDs with uniform interdot tunnel coupling $\tau$ at exact resonance when $\varepsilon\left(e_{1}\right)=\ldots=\varepsilon\left(e_{N}\right)$. The dependencies of $U_{q}\left(e_{k}\right)$ on the AS QD positions are presented in figure 2. Hereafter, we shall work with the effective atomic units $1 \mathrm{au}=R y^{*}=m^{*} R y / m_{e} \varepsilon^{2}$ for the energy, $1 \mathrm{au}=a_{B}^{*}=m_{e} \varepsilon a_{B} / m^{*}$ for the length, and $1 \mathrm{au}=\hbar / R y^{*}$ for the time, where $R y$ is the Rydberg energy, $a_{B}$ is the Bohr radius, $m_{e}$ is the free electron mass, $m^{*}$ is the effective electron mass, $\varepsilon$ is the dielectric constant, and $\hbar$ is the Planck constant. Note that the parameters indicated in figure 2 and used throughout the paper correspond to realistic GaAs QD system $\left(R y^{*}=6 \mathrm{meV}, a_{B}^{*}=10 \mathrm{~nm}\right)$.

The voltage-controlled energy shifts are parameterized by the expression $\delta U_{q^{\prime}}\left(e_{k}\right)=$ $-U_{q^{\prime}}\left(e_{k}\right)\left[\delta V / U_{0}\right]$, so that the external voltage compensates the electrostatic shift $U_{q^{\prime}}\left(e_{k}\right)$ of the energy level in the AS QD $A_{k}$ when $\delta V$ approaches $U_{0}$. Direct numerical diagonalization of the Hamiltonian, Eq. (1), results in two groups of two-electron eigenstates each corresponding to the qubit's localization in one of two logical states. The basis states $\left|g_{1}, 0\right\rangle,\left|g_{N}, 0\right\rangle,\left|g_{1}, 1\right\rangle$, and $\left|g_{N}, 1\right\rangle$ are the eigenstates of the Hamiltonian since we neglect the tunnel coupling between the AS ground states $\left|g_{1}\right\rangle$ and $\left|g_{N}\right\rangle$. Their energies $E\left(g_{1}, 0\right)=\varepsilon\left(g_{1}\right)+\varepsilon_{0}+U_{0}^{\prime}\left(g_{1}\right), E\left(g_{N}, 0\right)=\varepsilon\left(g_{N}\right)+\varepsilon_{0}+U_{0}^{\prime}\left(g_{N}\right)$, $E\left(g_{1}, 1\right)=\varepsilon\left(g_{1}\right)+\varepsilon_{1}+U_{1}^{\prime}\left(g_{1}\right)$, and $E\left(g_{N}, 1\right)=\varepsilon\left(g_{N}\right)+\varepsilon_{1}+U_{1}^{\prime}\left(g_{N}\right)$ depend on the qubit state, the external voltage, and the structure geometry. The excited eigenstates of the probe electron may be represented as the normalized superpositions $|m, q\rangle=\sum_{k=1}^{N} C_{m, k, q}\left|e_{k}, q\right\rangle$, where $m=1-N$ and $q=0,1$. Since we shall be interested in the PET between the AS ground states $\left|g_{1}\right\rangle$ and $\left|g_{N}\right\rangle$ localized in the AS QDs $A_{1}$ and $A_{N}$ through the resonant temporal population of one of the excited states $|m, q\rangle$, it is important to know the superposition coefficients $C_{m, 1, q}$ and $C_{m, N, q}$ reflecting the weights of that transport state in both edge AS QDs. Figures 3 (a) and 3 (b) illustrate the dependencies of absolute values of those coefficients for the states from the central part of excited subband $\left(8 \leq m \leq 13, N=20\right.$ ) on the parameter $\delta V / U_{0}$ when the compensating voltages remove the electrostatic shifts corresponding to the qubit state "one" $\left(q^{\prime}=1\right)$. The eigenenergies $E(m, q)$ are shown in figure 3 (c). All structure 
parameters are those given in Fig. 2. As it is observed from figures. $3(\mathrm{a}-\mathrm{c})$, the interaction of the AS with the qubit does not change considerably the resonant character of single-electron tunneling in the AS for both $q=0$ and $q=1$ cases for a given set of parameters bringing about almost uniform shift of one excited subband relative to another. The weight coefficients converge to their unperturbed values for $q=1$ at the point $\delta V=U_{0}$ where one has $\left|C_{m, 1,1}\right|=\left|C_{m, N, 1}\right|=\left|C_{N-m+1,1,1}\right|=\left|C_{N-m+1, N, 1}\right|$. For $q=0$ a very close coefficient behavior is seen at $\delta V \approx 0.4 U_{0}$, however, with some deviations from the symmetric picture demonstrated by the plots in the case of $q=1$. We have revealed that the states lying in the middle of the excited subband conserve their hybridized structure in the range $0 \leq \delta V \leq 2 U_{0}$, whereas the edge subband states (not shown) are very sensitive to the external voltage. In particular, the tunneling collapse described above manifests itself in that the edge subband state with $m=1$ ( $m$ $=N)$ splits off the subband and transforms at quite large positive (negative) values of $\delta V\left(|\delta V| \geq 3 U_{0}\right)$ into the isolated state of the QD $A_{N}$ with $\left|C_{1, N, q}\right| \approx 1\left(\left|C_{N, N, q}\right| \approx 1\right)$. The influence of the electrostatic interaction between the probe and qubit electrons on the AS spectral properties becomes stronger with the decrease in $r_{0}$ and/or with the increase in $L$.

\subsection{The PET and qubit phase shift in three-level approximation}

Next we demonstrate how to implement the PET between the ground states $\left|g_{1}\right\rangle$ and $\left|g_{N}\right\rangle$ of the edge AS QDs $A_{1}$ and $A_{N}$ given that the qubit is in one of its logical states, say, in the state $|1\rangle$. Furthermore, we shall show that the PET from $\left|g_{1}\right\rangle$ to $\left|g_{N}\right\rangle$ followed by the reversal PET from $\left|g_{N}\right\rangle$ to $\left|g_{1}\right\rangle$ can produce the phase shift between logical qubit states.

Initially, the probe electron resides in the ground state $\left|g_{1}\right\rangle$ of the QD $A_{1}$, whereas the state $|q\rangle=c_{0}|0\rangle+c_{1}|1\rangle$ of the qubit is arbitrary. Therefore, our two-electron system is characterized by the state vector

$$
\left|\Psi_{i}\right\rangle=\left\{c_{0} \exp \left[-i E\left(g_{1}, 0\right) t\right]|0\rangle+c_{1} \exp \left[-i E\left(g_{1}, 1\right) t\right]|1\rangle\right\}\left|g_{1}\right\rangle .
$$

Note, that $U_{0}\left(e_{1}\right) \approx U_{1}\left(e_{1}\right)$ (see figure 2) and $E\left(g_{1}, 0\right)-E\left(g_{1}, 1\right) \approx \varepsilon_{0}-\varepsilon_{1}$, therefore, the phase difference between two state components in Eq. (4) is mostly defined by the difference between qubit logical state energies. When the compensating voltages $\left\{\delta U_{1}\left(e_{k}\right)\right\}_{k=1}^{N}$ are turned on, the excited two-electron eigenstates $|m, 1\rangle(m=1-N)$ transform at $\delta V=U_{0}$ into the unperturbed eigenstates with high transport properties. According to Refs. [18] - [22], to attain the indirect resonant PET (now conditioned by the qubit state $|1\rangle)$ between the states $\left|g_{1}\right\rangle$ and $\left|g_{N}\right\rangle$ localized in the QD $A_{1}$ and $A_{N}$, one has to irradiate the AS either by a pair of resonant laser pulses if $\varepsilon\left(g_{1}\right) \neq \varepsilon\left(g_{N}\right)$ (asymmetrical case) or by single pulse if $\varepsilon\left(g_{1}\right) \approx \varepsilon\left(g_{N}\right)$ (symmetrical case). Below we consider the asymmetrical case, where the ground-state energies of edge QDs substantially differ from each other. The pulse frequencies $\omega_{0}$ and $\omega_{1}$ match the resonant transition frequencies $\omega\left(g_{1}, r, 1\right)=E(r, 1)-E\left(g_{1}, 1\right)$ and $\omega\left(g_{N}, r, 1\right)=E(r, 1)-E\left(g_{N}, 1\right)$ between the localized states $\left|g_{1}, 1\right\rangle$ and $\left|g_{N}, 1\right\rangle$ and some excited (transport) state $|r, 1\rangle$ 
[for concreteness, $\omega_{0}=\omega\left(g_{1}, r, 1\right)$ and $\left.\omega_{1}=\omega\left(g_{N}, r, 1\right)\right]$. Further, the pulse strengths $E_{0}$ and $E_{1}$ have to be chosen so that the absolute values of the coupling coefficients $\lambda\left(g_{1}, r, 1\right)=E_{0} d\left(g_{1}, r, 1\right) / 2$ and $\lambda\left(g_{N}, r, 1\right)=E_{1} d\left(g_{N}, r, 1\right) / 2$, where $d\left(g_{1}, r, 1\right)$ and $d\left(g_{N}, r, 1\right)$ are the corresponding matrix elements of optical dipole transition, be equal to each other, i. e., $\left|\lambda\left(g_{1}, r, 1\right)\right|=\left|\lambda\left(g_{N}, r, 1\right)\right| \equiv \lambda$. It is also important that only the state $|r, 1\rangle$ from the AS excited subband is to be selectively populated during the pulse action. It means that the differences $\Delta(r, r \pm 1,1)=E(r, 1)-E(r \pm 1,1)$ between the energy of transport state $|r, 1\rangle$ and the energies of the nearest states $|r \pm 1,1\rangle$ must be large compared with the coupling coefficient $\lambda:|\Delta(r, r \pm 1,1)| \gg \lambda$. Besides, we should prevent the population of states from another excited subband corresponding to the qubit state $|0\rangle$, by careful choice of the structure and pulse parameters (see Sec. 2.3). Finally, it is assumed that $\left|\varepsilon\left(g_{1}\right)-\varepsilon\left(g_{N}\right)\right| \gg \lambda$ so that each pulse drives only its own transition. (This requirement is relevant only in asymmetrical case.) As soon as all above conditions are satisfied, only three states, viz. $\left|g_{1}, 1\right\rangle,\left|g_{N}, 1\right\rangle$, and $|r, 1\rangle$, are optically active, and the effective three-level Hamiltonian describing the resonant transfer process under the rotating-wave approximation $\left(\lambda \ll \omega_{0}, \omega_{1}\right)$ takes the form

$$
H_{R W A}=\lambda\left(g_{1}, r, 1\right)\left|g_{1}, 1\right\rangle\left\langle r, 1\left|+\lambda\left(g_{N}, r, 1\right)\right| g_{N}, 1\right\rangle\langle r, 1|+\text { h.c. }
$$

In the rotating frame, the coherent evolution of the state vector $\left|\Psi_{\Lambda}\right\rangle=a_{1,1}\left|g_{1}, 1\right\rangle+$ $a_{N, 1}\left|g_{N}, 1\right\rangle+\tilde{a}_{r, 1}|r, 1\rangle$, spanned by those states, is governed by the non-stationary Schrödinger equation $i \partial\left|\Psi_{\Lambda}\right\rangle / \partial t=H_{R W A}\left|\Psi_{\Lambda}\right\rangle$. Let the pulses be switched on at $t$ $=0$. For the initial condition $\left|\Psi_{\Lambda}(0)\right\rangle=\left|g_{1}, 1\right\rangle$, the solution of this equation is well known (see, e. g., Ref. [20]) and describes the three-level Rabi oscillations:

$$
a_{1,1}=\cos ^{2}\left(\Omega_{R} t\right), \quad a_{N, 1}=-\sin ^{2}\left(\Omega_{R} t\right), \quad \tilde{a}_{r, 1}=-\frac{i}{\sqrt{2}} \sin \left(2 \Omega_{R} t\right),
$$

where $\Omega_{R}=\lambda / \sqrt{2}$ is the Rabi frequency. Thus, the complete PET from the ground state of edge AS QD $A_{1}$ to the ground state of edge AS QD $A_{N}$ takes place in times $T_{n}=(\pi / 2+\pi n) / \Omega_{R}$, where $n=0,1,2, \ldots$ In what follows, we shall consider the shortest time $T_{0}=\pi / 2 \Omega_{R}$ as the PET time.

At the end of the optical transfer $\left(t=T_{0}\right)$, the state vector of our system in the laboratory frame transforms into

$$
|\Psi\rangle=c_{0} \exp \left[-i E\left(g_{1}, 0\right) T_{0}\right]|0\rangle\left|g_{1}\right\rangle-c_{1} \exp \left[-i E\left(g_{N}, 1\right) T_{0}\right]|1\rangle\left|g_{N}\right\rangle .
$$

After the time $\tau_{0}$ during which no pulses act on the AS, we drive the probe electron from the state $\left|g_{N}\right\rangle$ back to the state $\left|g_{1}\right\rangle$ in the same manner and then turn off the compensating voltages. As a result, the component of the two-electron state corresponding to the qubit state $|1\rangle$ acquires an additional phase $\theta=\delta E\left(T_{0}+\tau_{0}\right)$ [in comparison with the free evolution case of Eq. (4)], where $\delta E=E\left(g_{N}, 1\right)-$ $\left.E\left(g_{1}, 1\right)\right|_{\delta V=U_{0}}=\varepsilon\left(g_{N}\right)-\varepsilon\left(g_{1}\right)$, so that for $t \geq 2 T_{0}+\tau_{0}$ the state vector reads

$$
\left|\Psi_{f}\right\rangle=\left\{c_{0} \exp \left[-i E\left(g_{1}, 0\right) t\right]|0\rangle+c_{1} \exp (-i \theta) \exp \left[-i E\left(g_{1}, 1\right) t\right]|1\rangle\right\}\left|g_{1}\right\rangle \text {. }
$$

For $\theta=\pi(2 m+1), m=0, \pm 1, \pm 2, \ldots$ the above operations amount to the phase shift of the qubit logical state $|1\rangle$ by $\pi$ in the qubit frame - i. e., $|q\rangle\left|g_{1}\right\rangle \rightarrow(Z|q\rangle)\left|g_{1}\right\rangle$, where 
$Z=|0\rangle\langle 0|-| 1\rangle\langle 1|$. It is worth noting that given phase shift is defined by the difference between energies of AS ground states rather than qubit states. If $\varepsilon\left(g_{N}\right)-\varepsilon\left(g_{1}\right)=0$ (e. g., when QD $A_{1}$ and $A_{N}$ are identical), the phase difference between degenerate states $\left|g_{1}\right\rangle$ and $\left|g_{N}\right\rangle$ is not accumulated and we need another approach to produce a phase shift. For example, by varying the compensating voltage on the QD $A_{N}$ (after the PET $\left|g_{1}\right\rangle \rightarrow\left|g_{N}\right\rangle$ now driven by single pulse have been completed), it is possible to generate required phase shift $\theta=\int_{0}^{\tau_{0}} \delta \varepsilon^{\prime}\left(t^{\prime}\right) d t^{\prime}$ by appropriate choice of the voltagecontrolled energy shift $\delta \varepsilon^{\prime}\left(t^{\prime}\right)$ of the state $\left|g_{N}\right\rangle$ and the variation time $\tau_{0}$. The time $\tau_{0}$ is thus exploited as the additional independent parameter to control the qubit phase in both asymmetrical and symmetrical cases.

\subsection{Numerical simulations of the PET in 2(N+2)-level case}

The formulas (5) and (6) describe an idealized three-level evolution through which PET is realized with the probability $p_{N, 1}\left(T_{0}\right)=\left|a_{N, 1}\left(T_{0}\right)\right|^{2}=1$. However, other excited states $|m, 1\rangle$ with $m \neq r$ (especially those nearest to the transport state) participate electron dynamics resulting in some decrease of $p_{N, 1}\left(T_{0}\right)$. As it was shown in Ref. [24], the main reason for such behavior is non-correlated off-resonant excitations of those states during the pulse action. The PET probability $p_{N, 1}\left(T_{0}\right)$ accounting for these processes is now given by the expression 24 ]

$$
\begin{aligned}
& p_{N, 1}\left(T_{0}\right) \approx 1-\sum_{m \neq r} f_{m}, \\
& f_{m}=\left[2 \lambda\left(g_{1}, m, 1\right) / \Delta(m, r, 1)\right]^{2} \sin ^{2}\left[\pi \Delta(m, r, 1) / 2 \sqrt{2} \lambda\left(g_{1}, r, 1\right)\right],
\end{aligned}
$$

where $\Delta(m, r, 1)=E(m, 1)-E(r, 1)$ and we require that $\left|\lambda\left(g_{1}, m, 1\right)\right| \approx\left|\lambda\left(g_{N}, m, 1\right)\right|$ for arbitrary $m$. Moreover, despite of inefficiency of the PET between ground states $\left|g_{1}\right\rangle$ and $\left|g_{N}\right\rangle$ of the edge AS QDs for two-electron state component in Eq. (4) corresponding to the qubit state "zero", off-resonant excitations from the ground state $\left|g_{1}, 0\right\rangle$ to the states $|m, 0\rangle(m=1-N)$ may also happen. It may amount to noticeable reduction of the probability $p_{1,0}\left(T_{0}\right)$ of probe electron to stay in the state $\left|g_{1}, 0\right\rangle$ at the pulse end.

The PET optimization is thus considered as the search of the pulse and structure parameters for which both $p_{N, 1}\left(T_{0}\right)$ and $p_{1,0}\left(T_{0}\right)$ are as large as possible while the transfer time $T_{0}$ is rather short. In particular, as we have mentioned before, it requires the careful choice of transport state $|r, 1\rangle$ that has to possess large coupling coefficient $\lambda\left(g_{1}, r, 1\right)$ and be well separated from neighboring states. According to the results of the work [24], in a quasi-one-dimensional structure the states belonging to the central part of excited subband are the best candidates for this purpose. If the QD number $N$ is even, they are the states with indices $r=N / 2$ or $r=N / 2+1$, whereas for odd $N$ this is the unpaired state with the index $r=(N+1) / 2$.

In what follows, we consider a general AS+qubit state in laboratory frame

$$
|\Psi\rangle=\sum_{q=0,1} c_{q}\left\{a_{1, q} e^{-i E\left(g_{1}, q\right) t}\left|g_{1}, q\right\rangle+a_{N, q} e^{-i E\left(g_{N}, q\right) t}\left|g_{N}, q\right\rangle+\sum_{m=1}^{N} \tilde{a}_{m, q} e^{-i E(m, q) t}|m, q\rangle\right\}
$$


as the superposition of $2(N+2)$ two-electron eigenstates at $\delta V=U_{0}, q^{\prime}=1$. Since we suppose that there are no transitions between logical qubit states during the PET in the AS, the subspaces $\left\{\left|g_{1}, 0\right\rangle,\left|g_{N}, 0\right\rangle,|m, 0\rangle_{m=1}^{N}\right\}$ and $\left\{\left|g_{1}, 1\right\rangle,\left|g_{N}, 1\right\rangle,|m, 1\rangle_{m=1}^{N}\right\}$, corresponding to different logical qubit states, are decoupled from each other and can be treated separately. To study coherent probe electron evolution in the AS under the influence of a pair of resonant square laser pulses polarized along the AS axis $x$, the non-stationary Schrödinger equation

$$
i \partial \mathbf{a}_{q} / \partial t=\boldsymbol{\Lambda}(q)\left[\cos \left(\omega_{0} t\right)+\cos \left(\omega_{1} t\right)\right] \mathbf{a}_{q}, \quad q=0,1,
$$

governing the probability amplitude vector $\mathbf{a}_{q}=\left(a_{1, q}, a_{N, q}, \tilde{a}_{1, q}, \ldots, \tilde{a}_{N, q}\right)^{T}$, is solved numerically for the initial condition $\mathbf{a}_{q}(0)=(1,0,0, \ldots, 0)^{T}$. Here the matrix $\boldsymbol{\Lambda}(q)$ with the entries $\Lambda_{k k^{\prime}}(q)=2 \lambda\left(k, k^{\prime}, q\right) \exp \left[-i \Delta\left(k, k^{\prime}, q\right) t\right]$ characterizes the optical dipole coupling strength for each transition between the two-electron eigenstates from given subspace, and indices $k$ and $k^{\prime}$ run over all of those states. The intersubband coupling coefficients between the ground states $\left|g_{1}, q\right\rangle,\left|g_{N}, q\right\rangle$ and an arbitrary excited state $|m, q\rangle$ are calculated within tight-binding model as

$$
\lambda\left(g_{1(N)}, m, q\right)=\varepsilon_{\text {field }} d\left(g_{1(N)}, m, q\right) / 2, d\left(g_{1(N)}, m, q\right)=C_{m, 1(N), q} d_{0},
$$

while the intrasubband coupling coefficients can be found from the expressions

$$
\begin{aligned}
& \lambda\left(g_{1(N)}, g_{1(N)}, q\right)=\mp \varepsilon_{\text {field }}(N-1) r_{c} / 4, \lambda\left(g_{1}, g_{N}, q\right)=0, \\
& \lambda(m, n, q)=-\varepsilon_{\text {field }} \sum_{k} C_{m, k, q}^{*} C_{n, k, q}[(N-1) / 2-k+1] r_{c} / 2,
\end{aligned}
$$

where $\varepsilon_{\text {field }}=e a_{B}^{*} E_{0} / R y^{*}$ is the field energy (actually, the dimensionless field strength), $d_{0}=\left\langle g_{1}|-x| e_{1}\right\rangle$ is the matrix element of optical dipole transition between the states $\left|g_{1}\right\rangle$ and $\left|e_{1}\right\rangle$ of isolated QD $A_{1}$, and the origin is placed at the axis $x$ in the center of the AS. Note, that in Eq. (11) we suppose the condition $E_{0}=E_{1}$, underlying the coupling coefficients symmetry $\left|\lambda\left(g_{1}, m, 1\right)\right|=\left|\lambda\left(g_{N}, m, 1\right)\right|$, to be fulfilled. One can derive Eqs. (12) and (13) from the optical dipole matrix element's definition substituting in it the superpositional form for an excited hybridized state $|m, q\rangle$. We neglect in Eq. (12) the interdot optical dipole transitions setting $\left\langle g_{1}|-x| e_{k \neq 1}\right\rangle \approx\left\langle g_{N}|-x| e_{k \neq N}\right\rangle \approx 0$ and express in Eq. (13) the level shifts as $\left\langle e_{k}|-x| e_{k}\right\rangle=-[(N-1) / 2-k+1] r_{c}$ and $\left\langle e_{k}|-x| e_{k^{\prime} \neq k}\right\rangle \approx 0$ (the latter are much smaller than those where $k=k^{\prime}$ and have been discarded). Though the intrasubband terms have large coupling values in comparison with the intersubband ones, their contribution into the dynamics is minor since they oscillate at very low frequencies too far from resonance with the driving pulses. The dipole approximation used in Eq. (5) and Eq. (11) holds if the effective AS length $l_{c}=(N-1) r_{c}$ is much smaller than the radiation wavelength $\lambda_{w}$. In our model $l_{c} \sim$ $10^{-6} \mathrm{~m}, \lambda_{w} \sim 10^{-4} \mathrm{~m}$ and, therefore, one has $l_{c} \ll \lambda_{w}$.

Setting $q=1$, we find from numerical solution of Eq. (11) the maximal PET probability $\max \left(p_{N, 1}\right)=p_{N, 1}\left(T_{0}\right)$ and corresponding transfer time $T_{0}$ as functions of $\varepsilon_{\text {field }}$ and then calculate the probability $p_{1,0}\left(T_{0}\right)=\left|a_{1,0}\left(T_{0}\right)\right|^{2}$. In figure 4 we show the dependency of $\max \left(p_{N, 1}\right)$ (filled circles represent numerical data and solid curve visualizes the approximation of Eq. (9)) as well as the dependencies of $p_{1,0}\left(T_{0}\right)$ vs the 
field energy $\varepsilon_{\text {field }}$ for three values of $r_{0}: p_{1,0}$ for $r_{0}=2, p_{1,0}^{\prime}$ for $r_{0}=3$, and $p_{1,0}^{\prime \prime}$ for $r_{0}=4$ (solid lines with open squares). Here $d_{0}=0.22\left(1 \mathrm{au}=e a_{B}^{*}\right), r=10$ (the index of the transport state), $\omega_{0}=15.083, \omega_{1}=18.417$ and the remaining parameters are the same as above. We see that numerical and analytical data for $\max \left(p_{N, 1}\right)$ correlate well with each other thus confirming our arguments used to justify the application of Eq. (9). From the other hand, it is rather difficult to construct a reliable approximation for the probability $p_{1,0}$. If $L \gg r_{0}$, the oscillations of this function can be satisfactory reproduced by the off-resonant solution for two-level system involving the state $\left|g_{1}, 0\right\rangle$ and excited state $|m, 0\rangle$ closest to the state $|r, 1\rangle$. Otherwise, if $L \ll r_{0}$, the populations of both components of a two-electron state demonstrate very close behavior because now it is hard to distinguish between the qubit charge states and the selectivity requirement is violated. We are interested, however, in the situation where $L$ and $r_{0}$ are of the same order.

Comparing both plots we define the optimal values of $\varepsilon_{\text {field }}$ as an abscises of the graph points where the probabilities simultaneously achieve their maxima. It is directly observed from figure 4 that it takes place in the interval around $\varepsilon_{\text {field }}=0.08\left(E_{0} \approx\right.$ $500 \mathrm{~V} / \mathrm{cm}$ for GaAs) where the probabilities of interest are $\max \left(p_{N, 1}\right)=0.9989, p_{1,0}$ $=0.996$ at $\varepsilon_{\text {field }}=0.077$ and $\max \left(p_{N, 1}\right)=0.9955, p_{1,0}^{\prime \prime}=0.9974$ at $\varepsilon_{\text {field }}=0.08$. The plot illustrating the transfer time $T_{0}$ vs the field energy $\varepsilon_{\text {field }}$ (figure 5 ) helps us to find corresponding values of $T_{0}: T_{0}=846$ and $T_{0}=833$, respectively. For GaAs one has 1 au $=0.11 \mathrm{ps}$ and the transfer time is about $85 \mathrm{ps}$ (that is by an order of magnitude smaller than coherence time expected in GaAs nanostructures). Note, that for field energies $\varepsilon_{\text {field }} \geq 0.12$ the PET probability substantially reduces.

Let us summarize the results obtained in this Section. We have observed that conditional PET in a quasilinear QD chain between the edge QDs $A_{1}$ and $A_{N}$ can be carried out with high accuracy in relatively short times. If the probe electron is then returned back to its initial state (viz., the ground state of the QD $A_{1}$ ), the phase operation is performed on the qubit positioned near QD $A_{N}$. From the other hand, if our quasilinear chain is a part of complex AS (the case considered below) and the QD $A_{N}$ belongs also to the adjacent AS part, the PET between those AS parts becomes conditioned by the state of the qubit attached to the QD $A_{N}$. Therefore, the probe electron can be transferred from the QD $A_{1}$ via the AS to any other qubit provided that this first (control) qubit is in the state "one". Thus, it makes possible to implement the controlled-phase operation on given pair of (remote) qubits.

In general, to perform a phase shift by an angle $\theta$ on the target qubit provided that the control qubit is in the state "one", we have to implement conditional PET from initial QD of the structure to the QD positioned near control qubit (if and only if its state is "one"). Secondly, the conditional transfer from this near-to-control-qubit QD to the QD positioned near target qubit (again, if and only if its state is "one") should be carried out. Therefore, if the two-qubit state is $|11\rangle$, phase is accumulated in target qubit according to the scenario given in this Section. If the two-qubit state isn't $|11\rangle$, the probe electron doesn't go to target qubit and, therefore, phase isn't accumulated. It 
is equivalent to the transition from two-qubit state $|a b\rangle$ to state $[\exp (i \theta)]^{a b}|a b\rangle$, where $a, b=(0,1)$.

\section{Nine-qubit Shor encoding and error syndrome measurement}

It is known that any realistic quantum computational scheme should be protected from quantum errors caused by the environmental decoherence and the external control imperfections. For this purpose, one may use the quantum error correction codes converting individual qubit state into the specific entangled state of several qubits that, after decoding, restores initial single qubit state (see, e.g., the book of Nielsen and Chuang [1] for detail). In this Section we present the algorithm realizing the nine-qubit quantum error correction code developed by Shor [23].

\subsection{Auxiliary structure for Shor encoding algorithm}

The encoding procedure requires us to arrange the qubits in such a way that the interactions between them can be switched on/off on demand in controllable manner. Making use of the results of Sec. 2, we propose the following model of auxiliary structure to mediate the interqubit coupling and to assist the entanglement generation needed for the implementation of Shor encoding (see figure 6). Such an AS incorporates three branches $T, T^{\prime}$, and $T^{\prime \prime}$ each represented by a T-shaped QD structure. The QD $A_{1}$ enters all branches as their common first QD and contains the probe electron in its ground state $\left|g_{1}\right\rangle$ at the beginning of encoding procedure. Consider in detail the upper branch $T$ that is attached to the qubit $q_{A}$ whose state we are going to encode. Its vertical part replicates the quasilinear AS examined in Sec. 2. The QD $A_{N}$ positioned near the central (control) qubit $q_{A}$ is used not only for operations on that qubit but also for operations, conditioned by the state "one" of the qubit $q_{A}$, on two side (target) qubits $q_{B}$ and $q_{C}$ placed near the QDs $B_{N}$ and $C_{N}$. Thus the QD $A_{N}$ serves as the common first $\mathrm{QD}$ for two quasilinear substructures with the symmetry axis perpendicular to the axis $x$. The parameters of four QDs $A_{1}, A_{N}, B_{N}$, and $C_{N}$ are chosen so that their ground state energies satisfy following relations: $\varepsilon\left(g_{1}\right) \neq \varepsilon\left(g_{N}\right) \neq \varepsilon\left(g_{B_{N}}\right)$ and $\varepsilon\left(g_{B_{N}}\right)=\varepsilon\left(g_{C_{N}}\right)$. Other two branches $T^{\prime}$ and $T^{\prime \prime}$ are obtained from the branch $T$ through in-plane clockwise rotations around the center of the QD $A_{1}$ by the angles $2 \pi / 3$ and $4 \pi / 3$, respectively. We see that each branch mediates the interaction between the probe electron and a cluster of three spatially separated charge qubits. The tunnel couplings between the branches are controlled by the gates $G, G^{\prime}$, and $G^{\prime \prime}$. When a negative voltage is applied to one of those gates, the potential barrier separating the QD $A_{1}$ from corresponding branch grows exponentially, and the electron tunneling across the barrier quickly falls. As the calculations show the decrease of tunneling matrix element $\tau$ between two neighboring QDs by two orders of magnitude is enough to treat those QDs as isolated from each other. We shall consider each blockage gate to be either opened (no voltage) or closed (the voltage sufficient for tunneling's suppression is turned on). 
The AS parameters are taken so that to neglect the differences between the energies of all possible charge configurations in the cluster. Those energy differences can be minimized by appropriate choice of the QD number and/or the interdot distances in each branch. The cluster qubits are thus considered relative each other regardless their spatially distributed structure and, consequently, without any conditionality between them that might be caused by various configurations of the electron positions in the DQD structures. The spatial addressability of cluster qubits (understood here as the possibility of the state rotation of a chosen cluster qubit(s) without affecting the neighboring ones) is achieved due to the frequency and polarization selectivity of resonant pulses.

\subsection{Nine-qubit encoding scheme}

Let all qubits except the first qubit $q_{A}$ be initialized. We shall work in the qubit reference frame so that the phase multipliers arising from the qubit state energy differences will be omitted. We use the notation $\left|q_{A} q_{B} q_{C}\right\rangle=\left|q_{A}\right\rangle\left|q_{B}\right\rangle\left|q_{C}\right\rangle\left(q_{A}, q_{B}, q_{C}=0,1\right)$ for the threequbit basis state of the cluster coupled with the upper QD branch $T$. Similar notations for the three-qubit states of remaining two clusters attached to the QD branches $T^{\prime}$ and $T^{\prime \prime}$ are supplied with prime and double prime, respectively. In order to transform the AS+qubits state $\left|\Psi_{i}\right\rangle=\left[c_{0}|0\rangle+c_{1}|1\rangle\right]|00\rangle|000\rangle^{\prime}|000\rangle^{\prime \prime}\left|g_{1}\right\rangle$ into the encoded state

$$
\left|\Psi_{f}\right\rangle=\left[c_{0}\left|0_{S}\right\rangle+c_{1}\left|1_{S}\right\rangle\right]\left|g_{1}\right\rangle
$$

where $\left|0_{S}\right\rangle\left(\left|1_{S}\right\rangle\right)=2^{-3 / 2}[|000\rangle \pm|111\rangle]\left[|000\rangle^{\prime} \pm|111\rangle^{\prime}\right]\left[|000\rangle^{\prime \prime} \pm|111\rangle^{\prime \prime}\right]$ is the Shor code state (the code word) [23] corresponding to the single qubit state $|0\rangle(|1\rangle)$, we need to complete the following set of operations on the qubits and the AS.

According to standard decomposition of the encoding procedure into the set of single- and two-qubit operations [1], one initially should perform two controlled-NOT (CNOT) operations where qubit $q_{A}$ acts as control qubit whereas qubits $q_{A}^{\prime}$ and $q_{A}^{\prime \prime}$ are used as target qubits. Given operations entangle different clusters. Next, the Hadamard rotations are performed on each of those qubits. Finally, two CNOT operations are to be organized inside each of three clusters. In this case, central cluster qubit $q_{A}\left(q_{A}^{\prime}, q_{A}^{\prime \prime}\right)$ functions as the control qubit whereas corresponding side cluster qubits are target ones. Those operations entangle the qubits belonging to given cluster. What should we do to implement all of these steps using the AS shown in figure 6 ?

3.2.1. Entanglement between the clusters We perform the CNOT operation on the qubits $q_{A}$ and $q_{A}^{\prime}$ in following manner. Firstly, we have to transfer the probe electron from the ground state $\left|g_{1}\right\rangle$ of the central AS QD $A_{1}$ to the ground state $\left|g_{N}\right\rangle$ of the QD $A_{N}$ provided that the state of qubit $q_{A}$ is "one". For this purpose, we create the effective quantum channel connecting the QDs $A_{1}$ and $A_{N}$ via the probe electron tunneling along vertical part of the branch $T$. The application of compensating voltages to the QDs $A_{k}(k=1-\mathrm{N})$ amounts to the formation of the quasilinear QD structure with high transport properties, as it was described in Sec. 2.1. Note that apart from 
the electrostatic interaction between the probe electron and the electron bound in the qubit $q_{A}$, Eq. 3, the energy level shifts in the QDs $A_{k}$ are also affected by other qubits. It is essential, however, that required PET is to be conditioned by the state of the qubit $q_{A}$ only. To make transfer process insensitive to total charge state of remaining eight qubits, the maximal difference between interaction energies (here, the difference between interaction energies of the probe electron occupying the QD $A_{N}$ with those qubits all loaded either in the state "one" or "zero") should be much smaller than the coupling coefficient $\lambda$. In this case, the optical excitation of AS transport state will not depend on charge configuration of the qubits $q_{B}, \ldots, q_{C}^{\prime \prime}$. We have found that this condition is satisfied, e. g., for $\varepsilon_{\text {field }} \sim 0.1$ and $r_{c}=3$ when the QD number in the chain $A_{N}, \ldots$, $B_{N}\left(C_{N}\right)$ is greater than $18-20$. The contributions originated from those interactions to the compensating voltages can be expressed as averaged interaction energies for given QD.

Additionally, we close the gates $G^{\prime}$ and $G^{\prime \prime}$ in order to interrupt the tunnel coupling between the QD $A_{1}$ and the branches $T^{\prime}$ and $T^{\prime \prime}$. The blockage gates are used here to minimize the number of individual QD states that participate in the formation of excited subband. Such a reduction is accompanied with an increase in the spacings between nearest hybridized states and, therefore, it enhances the selectivity of the driving pulses. The numerical calculations illustrating the dependency of the probe electron energy spectrum on the external voltages indicate on the dissociation of total AS spectrum into the set of $N$ states delocalized over the quasilinear chain formed by QDs $A_{k}(k=1$ - N) and the states pertaining to other AS QDs. Residual tunnel coupling between QD $A_{N}$ and its neighbors in substructures attached to the qubits $q_{B}$ and $q_{C}$ has little effect on transport properties of our quasilinear AS and may be completely ruled out by the use of blockage gates analogous to those surrounding the QD $A_{1}$. In what follows, we shall not discuss in detail the formation of a quasilinear transport channel supposing that this task can be solved in all relevant cases.

As soon as the channel has been prepared, the PET can be attained according to the two-pulse resonant scheme since $\varepsilon\left(g_{1}\right) \neq \varepsilon\left(g_{N}\right)$ (see Sec. 2.2). At the end of the pulse action one has

$$
|\Psi\rangle=\left|\Psi_{0}\right\rangle+c_{1} \Phi\left(T_{0}\right)|100\rangle|000\rangle^{\prime}|000\rangle^{\prime \prime}\left|g_{N}\right\rangle
$$

where the state vector component corresponding to the qubit state "zero" is denoted by $\left|\Psi_{0}\right\rangle=c_{0}|000\rangle|000\rangle^{\prime}|000\rangle^{\prime \prime}\left|g_{1}\right\rangle$, and $\Phi\left(T_{0}\right)$ is the phase multiplier. This form of the state vector establishes the conditional probe electron evolution needed to construct at following steps the controlled-phase operations on two pairs of qubits, $\left(q_{A}, q_{A}^{\prime}\right)$ and $\left(q_{A}\right.$, $\left.q_{A}^{\prime \prime}\right)$, where $q_{A}$ plays role of control qubit.

Secondly, the Hadamard rotation $\mathrm{H}\left(q_{A}^{\prime}\right)=2^{-1 / 2}[|0\rangle+|1\rangle]\left\langle 0\left|+2^{-1 / 2}[|0\rangle-|1\rangle]\langle 1|\right.\right.$ is implemented on the qubit $q_{A}^{\prime}$ :

$$
|\Psi\rangle=\mathrm{H}\left(q_{A}^{\prime}\right)\left|\Psi_{0}\right\rangle+c_{1} \Phi(t) 2^{-1 / 2}|100\rangle\left(|000\rangle^{\prime}+|100\rangle^{\prime}\right)|000\rangle^{\prime \prime}\left|g_{N}\right\rangle
$$

(The single-qubit Hadamard gate can be accomplished via optical technique described in Ref. [20].) Further, we should perform the phase operation $Z$ on the qubit $q_{A}^{\prime}$ via the 
PET between the ground states $\left|g_{N}\right\rangle$ and $\left|g_{N}^{\prime}\right\rangle$ of the AS QDs $A_{N}$ and $A_{N}^{\prime}$, conditioned by the qubit state $\left|q_{A}\right\rangle=|1\rangle$ (namely, the controlled-phase operation), without affecting the state component $\mathrm{H}\left(q_{A}^{\prime}\right)\left|\Psi_{0}\right\rangle$. To do this, the gates $G$ and $G^{\prime}$ are opened and two laser pulses with equal strengths and frequencies, polarized along axes $x$ and $x^{\prime}$, act upon the AS. Optically active part of the AS is now presented by two identical quasilinear $\mathrm{QD}$ chains with the edge QDs $A_{N}$ and $A_{N}^{\prime}$. (Some specific features of excited state hybridization in the simplest planar artificial molecule composed of three disk QDs were analyzed in Ref. [19].) The pulse frequency matches the resonant frequency for optical transitions connecting the ground states $\left|g_{N}\right\rangle$ and $\left|g_{N}^{\prime}\right\rangle$ with the transport state. If the ground-state energies of QDs $A_{1}$ and $A_{N}\left(A_{N}^{\prime}\right)$ are substantially different from each other, the pulses do not address the ground state $\left|g_{1}\right\rangle$ of the central QD $A_{1}$. From other hand, the AS QDs $A_{N}$ and $A_{N}^{\prime}$ are identical, therefore, required phase shift has to be achieved through the voltage sweeping on the QD $A_{N}^{\prime}$ (see Sec. 2.2). After necessary voltage manipulations and probe electron's return into the ground state $\left|g_{N}\right\rangle$, the state vector becomes

$$
|\Psi\rangle=\mathrm{H}\left(q_{A}^{\prime}\right)\left|\Psi_{0}\right\rangle+c_{1} \Phi(t) 2^{-1 / 2}|100\rangle\left(|000\rangle^{\prime}-|100\rangle^{\prime}\right)|000\rangle^{\prime \prime}\left|g_{N}\right\rangle .
$$

As it is clearly seen, above operations are equivalent to CNOT operation on the pair of qubits $q_{A}$ and $q_{A}^{\prime}$ followed by Hadamard rotation of the target qubit $q_{A}^{\prime}$.

Next we repeat above procedure for the qubit $q_{A}^{\prime \prime}$ obtaining

$$
|\Psi\rangle=\mathrm{H}\left(q_{A}^{\prime \prime}\right) \mathrm{H}\left(q_{A}^{\prime}\right)\left|\Psi_{0}\right\rangle+c_{1} \Phi(t) 2^{-1}|100\rangle\left(|000\rangle^{\prime}-|100\rangle^{\prime}\right)\left(|000\rangle^{\prime \prime}-|100\rangle^{\prime \prime}\right)\left|g_{N}\right\rangle
$$

and then transfer the probe electron from the state $\left|g_{N}\right\rangle$ back to the state $\left|g_{1}\right\rangle$. Performing the Hadamard rotation $\mathrm{H}\left(q_{A}\right)$ on the qubit $q_{A}$ we arrive at the state vector

$$
|\tilde{\Psi}\rangle=\left[c_{0}\left|\tilde{\Psi}_{0}\right\rangle+c_{1} \Phi\left(T_{1}\right)\left|\tilde{\Psi}_{1}\right\rangle\right]\left|g_{1}\right\rangle,
$$

where $\left|\tilde{\Psi}_{0(1)}\right\rangle=2^{-\frac{3}{2}}(|000\rangle \pm|100\rangle)\left(|000\rangle^{\prime} \pm|100\rangle^{\prime}\right)\left(|000\rangle^{\prime \prime} \pm|100\rangle^{\prime \prime}\right)$. Note, that at this stage we have realized on the qubits $q_{A}, q_{A}^{\prime}$, and $q_{A}^{\prime \prime}$ the three-qubit Shor encoding scheme that is able to correct trivial phase error. It is easy to write the expression for the time $T_{1}$ required for above operations as $T_{1}=6 T_{0}+3 T_{\text {Had }}+2 \tau_{0}$, where we approximately set all transfer times to be equal to $T_{0}, T_{H a d}$ is the time reserved for Hadamard rotation, and $\tau_{0}$ is the voltage sweeping time. The phase $\theta\left(T_{1}\right)=\left[\varepsilon\left(g_{N}\right)-\varepsilon\left(g_{1}\right)\right]\left(T_{1}-T_{0}\right)$ accumulated during the process has to be equal to $2 \pi n$ ( $n$ is integer) in order to cancel the multiplier $\Phi\left(T_{1}\right)=\exp \left[-i \theta\left(T_{1}\right)\right]$. It may be attained through the appropriate choice of the time $T_{1}$.

3.2.2. Entanglement inside the clusters Finishing part of encoding algorithm consists in the implementation of CNOT operations on the target cluster qubits $q_{B}\left(q_{B}^{\prime}, q_{B}^{\prime \prime}\right)$ and $q_{C}\left(q_{C}^{\prime}, q_{C}^{\prime \prime}\right)$ with the control qubit $q_{A}\left(q_{A}^{\prime}, q_{A}^{\prime \prime}\right)$. Since $X=\mathrm{H} Z \mathrm{H}$, we perform initially the Hadamard rotation on one of target qubits, then carry out the phase operation $Z$, conditioned by the control qubit state "one", on that qubit and, as the final step, perform again the Hadamard rotation on the target qubit. This scheme is illustrated by evolution of three-qubit cluster state $\left|q_{A} q_{B} q_{C}\right\rangle$. The phase shift by $\pi$ of logical state 
"one" of target qubit $q_{B}\left(q_{C}\right)$ requires the conditional PET from the QD $A_{1}$ to the QD $A_{N}$ and then from the QD $A_{N}$ to the QD $B_{N}\left(C_{N}\right)$ and back. The main steps that bring about the CNOT implementation on the qubit $q_{B}$ are given below:

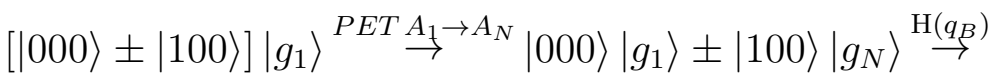

$$
\begin{aligned}
& |0\rangle[|0\rangle+|1\rangle]|0\rangle\left|g_{1}\right\rangle \pm|0\rangle[|0\rangle+|1\rangle]|0\rangle\left|g_{N}\right\rangle \stackrel{\text { PET } A_{N} \rightarrow B_{N} \rightarrow A_{N}}{\rightarrow} \\
& |0\rangle[|0\rangle+|1\rangle]|0\rangle\left|g_{1}\right\rangle \pm|0\rangle[|0\rangle-|1\rangle]|0\rangle\left|g_{N}\right\rangle \stackrel{\mathrm{H}\left(q_{B}\right)}{\rightarrow}|000\rangle\left|g_{1}\right\rangle \pm|110\rangle\left|g_{N}\right\rangle \text {. }
\end{aligned}
$$

[Here we omit in expressions of the state vector the phase multiplier $\Phi(t)$ and normalization coefficients. The qubit states of other two clusters remain unchanged and are not shown.] After the repeat of CNOT on the qubit $q_{C}$ and the return of probe electron into the state $\left|g_{1}\right\rangle$, the components of the nine-qubit state, Eq. (19), transforms into

$$
\left|\tilde{\Psi}_{0(1)}\right\rangle \rightarrow 2^{-\frac{3}{2}}(|000\rangle \pm|111\rangle)\left(|000\rangle^{\prime} \pm|100\rangle^{\prime}\right)\left(|000\rangle^{\prime \prime} \pm|100\rangle^{\prime \prime}\right) .
$$

This sequence of operations takes the time $T_{2}=6 T_{0}+4 T_{\text {Had }}+2 \tau_{0}$. Having performed the set of CNOT operations on remaining two qubit clusters $\left(q_{A}^{\prime}, q_{B}^{\prime}, q_{C}^{\prime}\right)$ and $\left(q_{A}^{\prime \prime}, q_{B}^{\prime \prime}, q_{C}^{\prime \prime}\right)$ we arrive at the desired encoded state, Eq. (14). The total time $T_{\text {tot }}$ of encoding algorithm's realization is now expressed by

$$
T_{\text {tot }}=T_{1}+3 T_{2}=24 T_{0}+15 T_{\text {Had }}+8 \tau_{0} .
$$

3.2.3. Time and probability estimations of encoding procedure Let us evaluate the time $T_{\text {tot }}$ using the value of $T_{0}$ calculated in Sec. 2.3. Setting $T_{\text {Had }} \sim \tau_{0} \sim 10 \mathrm{ps}$ (the value that is longer than it was taken in Ref. [20]) we find from Eq. (22) $T_{\text {tot }} \sim 2.3 \mathrm{~ns}$ at $\varepsilon_{\text {field }}=0.077$. We see that the transfer processes are the most prolonged part of the Shor encoding algorithm. Hence, the probability of successful encoding implementation can be roughly estimated as $p_{\text {Shor }} \sim \min \left[\left(p_{N, 1}\right)^{24},\left(p_{1,0}\right)^{24}\right]$ given that all other steps were performed without errors. It yields us the value $p_{\text {Shor }} \sim 0.91$ that is, of course, insufficient for large-scale fault-tolerant quantum computations. However, this estimation establishes the possibility to realize given scenario in proof-of-principle experiments. The way of

further optimization of the proposed algorithm includes the search of pulse and structure parameters that would provide an increase of the probability $p_{\text {Shor }}$ with simultaneous decrease of the time $T_{t o t}$.

\subsection{Error syndrome measurement}

It is known that with the help of nine-qubit Shor code one may, in principle, correct arbitrary single-qubit quantum errors occurring independently as long as an appropriate error syndrome measurement strategy exists. Here we demonstrate an algorithm correcting the $X$-type quantum error, viz., the environmentally induced qubit state inversion. Suppose that such an error has occurred in one of cluster qubits $q_{A}, q_{B}$, or $q_{C}$. In order to detect and correct it, one should perform the measurements of the observables $Z\left(q_{A}\right) Z\left(q_{B}\right)=[|00\rangle\langle 00|+| 11\rangle\langle 11|] \otimes I_{C}-[|01\rangle\langle 01|+| 10\rangle\langle 10|] \otimes I_{C}$ and 
$Z\left(q_{A}\right) Z\left(q_{C}\right)=[|00\rangle\langle 00|+| 11\rangle\langle 11|] \otimes I_{B}-[|01\rangle\langle 01|+| 10\rangle\langle 10|] \otimes I_{B}$ (see Ref. [1] for detail). If the results of both measurements are " $+1 "$, the three-qubit cluster state hasn't been damaged. If the measurement of $Z\left(q_{A}\right) Z\left(q_{B}\right)$ gives " $+1 "$ ("- 1 ") and the measurement of $Z\left(q_{A}\right) Z\left(q_{C}\right)$ gives "-1" $("+1 ")$, the state of the qubit $q_{C}\left(q_{B}\right)$ has been inverted. Finally, if both measurements amount to the value "-1", it indicates on the error in the qubit $q_{A}$. Since the corrupted qubit is identified, one has to perform $X$ rotation on that qubit to recover the encoded state [Eq. (14)]. The quantum phase error $Z$ that is unitarily equivalent to the inversion error $X$ can be corrected in the same manner. Note, that syndrome measurement enables us to define only the damaged qubit but not the qubit state, i. e., it conserves the entanglement.

We propose the syndrome measurement technique that is based upon the same principle as the encoding procedure. Again, the probe electron evolution in the AS conditioned by the states of tested qubits helps us to reveal the quantum error. Consider the AS composed of two identical quasilinear QD chains and used to measure the observable $Z\left(q_{A}\right) Z\left(q_{B}\right)$ (figure 7 ). The central QD $A_{0}$ belongs to both chains and contains the probe electron in its ground state $\left|g_{0}\right\rangle$ at the beginning of the syndrome measurement. Two edge QDs $A_{0}^{\prime}$ and $A_{0}^{\prime \prime}$ with ground states $\left|g_{0}^{\prime}\right\rangle$ and $\left|g_{0}^{\prime \prime}\right\rangle$ are placed near the qubits $q_{A}$ and $q_{B}$, respectively, and their single electron states are affected by qubit states. The QD parameters are chosen so that the energies $\varepsilon\left(g_{0}\right), \varepsilon\left(g_{0}^{\prime}\right)$, and $\varepsilon\left(g_{0}^{\prime \prime}\right)$ of QD ground states $\left|g_{0}\right\rangle,\left|g_{0}^{\prime}\right\rangle$, and $\left|g_{0}^{\prime \prime}\right\rangle$ are equal to each other, i. e., $\varepsilon\left(g_{0}\right)=\varepsilon\left(g_{0}^{\prime}\right)=\varepsilon\left(g_{0}^{\prime \prime}\right)$. The application of the set of voltages, compensating the electrostatic shifts of the AS QD energy levels when the qubits are in the state "one", results in three different types of energy spectrum. If both qubits are in the state "zero", an electron tunneling via the AS excited subband remains inefficient. If the state of one of the qubits is "one", the transport properties of the QD chain, attached to that qubit, are recovered. Finally, if both qubits are in the state "one", the excited states become delocalized over the whole AS. Next we irradiate the AS by laser pulse of the frequency that matches the resonant transition frequency for situation where only one qubit is in its state "one". The strength and polarization of the pulse have to be taken so that the dynamical properties of transitions $\left|g_{0}\right\rangle \rightleftarrows|r\rangle \rightleftarrows\left|g_{0}^{\prime}\right\rangle$ and $\left|g_{0}\right\rangle \rightleftarrows|r\rangle \rightleftarrows\left|g_{0}^{\prime \prime}\right\rangle$ connecting the transport state $|r\rangle$ and the ground states localized in the central and edge QDs will be equivalent. It means that the transition times and PET probabilities have to be the same for two possible charge configurations corresponding to that case. As soon as those requirements are met, the pulse will produce the three-level resonant dynamics described by Eqs. (6) giving rise to the PET between the state $\left|g_{0}\right\rangle$ and only one of states $\left|g_{0}^{\prime}\right\rangle$ and $\left|g_{0}^{\prime \prime}\right\rangle$ (of course, if only one of qubits is in the state "one"). Note, that in the case where both qubits are in the state "one", despite of high degree of hybridization of the AS excited states, the probability of probe electron excitation from the state $\left|g_{0}\right\rangle$ is very low because of the resonant transition frequency mismatch. As a result, the probe electron leaves the $\mathrm{AS} \mathrm{QD} A_{0}$ at the end of pulse only if the state of tested pair of qubits is $\left|q_{A} q_{B}\right\rangle=|01\rangle$ or $\left|q_{A} q_{B}\right\rangle=|10\rangle$. Thus, an information about the eigenvalues of syndrome operator $Z\left(q_{A}\right) Z\left(q_{B}\right)$ can be extracted from routine detection of the probe electron in the QD 
$A_{0}$ by measurement of current $I_{Q P C}$ via the quantum point contact QPC. The eigenvalue $+1(-1)$ thus corresponds to the presence (absence) of the probe electron in the QD $A_{0}$. If the measurement indicates on that the probe electron has left the QD $A_{0}$, the pulse is applied again to return it into the state $\left|g_{0}\right\rangle$, and the compensating voltages are switched off. It is very important that we cannot distinguish between two equivalent paths (otherwise the projective measurement of qubit state would be realized). From other hand, if one arranges driving pulse so that those transitions become different enough (e. g., polarizing the pulse along one of the QD branches), the setup shown in figure 7 may be used for standard projective measurement of a charge qubit state. Other five syndrome operators can be measured in similar way.

\section{Conclusion}

In our paper we have proposed the way of the entanglement engineering in quantumdot-based nanostructures. As an important practical application of the developed framework, we have considered (to our knowledge, for first time) the realization of the nine-qubit Shor encoding scheme in two-dimensional array of the semiconductor charge qubits. Highly-entangled Shor code states protecting the quantum information against an arbitrary single-qubit error are generated by means of appropriate optical and voltage manipulations on the combined system involving both qubits and the auxiliary structure. As it was shown, the PET (conditioned by qubit state) along the auxiliary structure together with single-qubit Hadamard rotations underly the encoding procedure. Numerical calculations confirm the possibility of successful implementation of the transfer protocol. In general, the indirect qubit-qubit coupling looks as the best solution to organize distributed quantum state manipulations in the charge qubit array. The described principle of entanglement production that makes use of an auxiliary system mediating the interactions among qubits can be also adopted for quantum information processing schemes with fully electrical control.

\section{References}

[1] Nielsen M A and Chuang I L 2000 Quantum Computation and Quantum Information (Cambrige: Cambrige University Press)

[2] Valiev K A 2005 Usp. Fiz. Nauk 1753 Valiev K A 2005 Phys. Usp. 481 (Engl. Transl.)

[3] Oliver W D, Yamaguchi F, and Yamamoto Y 2002 Phys. Rev. Lett. 88037901

[4] Fabian J and Hohenester U 2005 Phys. Rev. B 72 201304(R)

[5] Tsukanov A V 2005 Phys. Rev. A 72022344

[6] Contreras-Pulido L D and Rojas F 2008 Phys. Rev. A 77032301

[7] Qiao B, Ruda H E, and Wang J 2002 Journ. App. Phys. 912524

[8] DiVincenzo D P, Bacon D, Kempe J, Burkard G, and Whaley K B 2000 Nature 408339

[9] Tanamoto T and Fujita S 2005 Phys. Rev. B 72085335

[10] Reina J H and Johnson N F 2000 Phys. Rev. A 63012303

[11] de Pasquale F, Giorgi G, and Paganelli S 2004 Phys. Rev. Lett. 93120502

[12] de Visser R L and Blaauboer M 2006 Phys. Rev. Lett. 96246801 
[13] Weinstein Y S, Hellberg C S, and Levy J 2005 Phys. Rev. A 72020304

[14] Tanamoto T 2000 Phys. Rev. A 61022305

[15] Schirmer S G, Oi D K L, and Greentree A D 2005 Phys. Rev. A 71012325

[16] Fedichkin L, Yanchenko M, and Valiev K A 2000 Nanotechnology 11387

[17] Tsukanov A V and Valiev K A 2007 Mikroelektronika 3683

Tsukanov A V and Valiev K A 2007 Russian Microelectronics 3667 (Engl. Transl.)

[18] Openov L A 1999 Phys. Rev. B 608798

[19] Oh J H, Ahn D, and Hwang S W 2000 Phys. Rev. A 62052306

[20] Openov L A and Tsukanov A V 2004 Pis'ma Zh. Eksp. Teor. Fiz. 80572

Openov L A and Tsukanov A V 2004 JETP Lett. 80503 (Engl. Transl.)

[21] Paspalakis E, Kis Z, Voutsinas E, and Terzis A F 2004 Phys. Rev. B 69155316

[22] Tsukanov A V 2006 Phys. Rev. B 73085308

[23] Shor P W 1995 Phys. Rev. A 52 R2493

[24] Tsukanov A V 2008 J. Phys.: Condens. Matter 20315204 


\section{Figure captions}

Figure 1. (color online) Auxiliary structure formed by quasilinear chain of QDs (circles) and supplied with controlling gates (rectangles) contains a single probe electron in the ground state $\left|g_{1}\right\rangle$ of the QD $A_{1}$. The qubit (single-electron double quantum dot) is positioned at the right from the structure and loaded in the logical state "one". Below, the AS potential profile along the structure axis $x$ is shown schematically for the cases where the compensating voltages are switched either on (solid line) or off (dashed line).

Figure 2. (color online) Electrostatic shifts of the AS QD levels for two logical qubit states as a functions of QD index $k$ (QD position). Here $U_{1}\left(e_{1}\right)-U_{0}\left(e_{1}\right)=0.00082$ $\mathrm{au}, U_{1}\left(e_{N}\right)-U_{0}\left(e_{N}\right)=0.3 \mathrm{au}$, and $U_{1}\left(e_{N}\right)-U_{1}\left(e_{N-1}\right)=0.3 \mathrm{au}$.

Figure 3. (color online) Characteristics of the AS eigenspectrum vs the control voltage parameter $\delta V / U_{0}$. a) The absolute values of the weight coefficients $C_{m, 1, q}$ in the QD $A_{1}$. b) The absolute values of the of weight coefficients $C_{m, N, q}$ in the QD $A_{N}$. c) The excited AS eigenenergies $E(m, q)$ shifted by $\varepsilon\left(e_{1}\right)+\varepsilon_{q}$. Plots for $q=1(0)$ are presented by thick solid (thin dashed) curves. The point $\delta V=U_{0}$ is marked by the vertical dotted line.

Figure 4. (color online) Populations $\max \left(p_{N, 1}\right)$ and $p_{1,0}, p_{1,0}^{\prime}, p_{1,0}^{\prime \prime}$ vs the field energy $\varepsilon_{\text {field }}$.

Figure 5. (color online) Transfer time $T_{0}$ vs the field energy $\varepsilon_{\text {field }}$. Thin curve with filled circles shows the numerical data and solid curve visualizes the formula $T_{0}=\pi / 2 \Omega_{R}$.

Figure 6. (color online) Schematics of the auxiliary structure used in the Shor encoding implementation (see text).

Figure 7. (color online) Auxiliary structure proposed for error syndrome measurement on pair of qubits $q_{A}$ and $q_{B}$ (see text). 

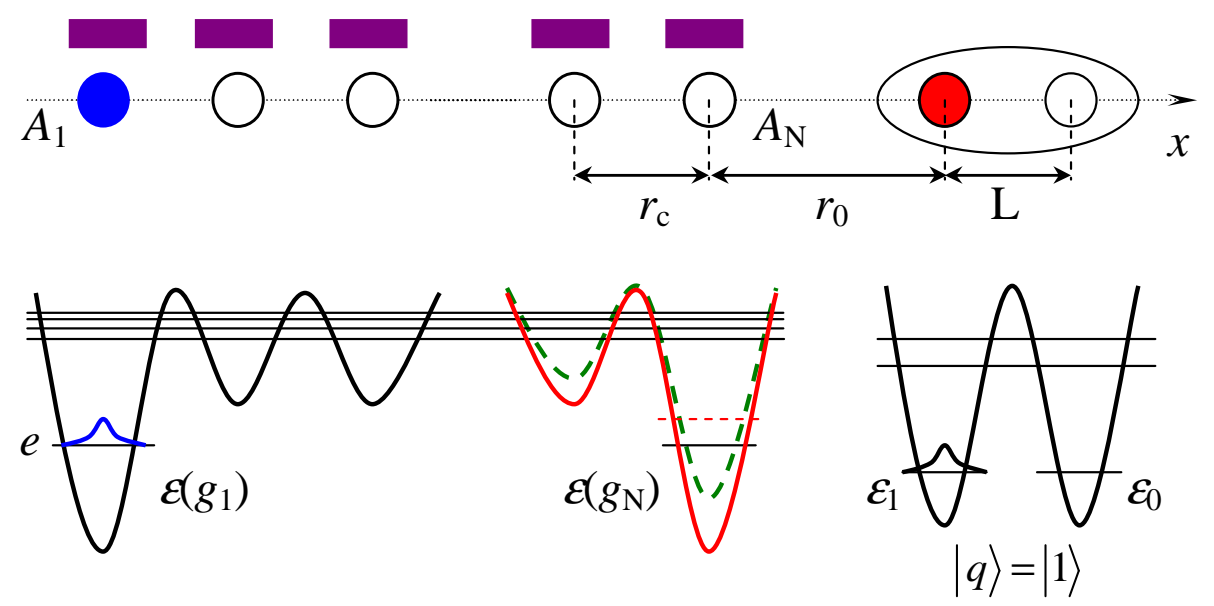

Figure 1

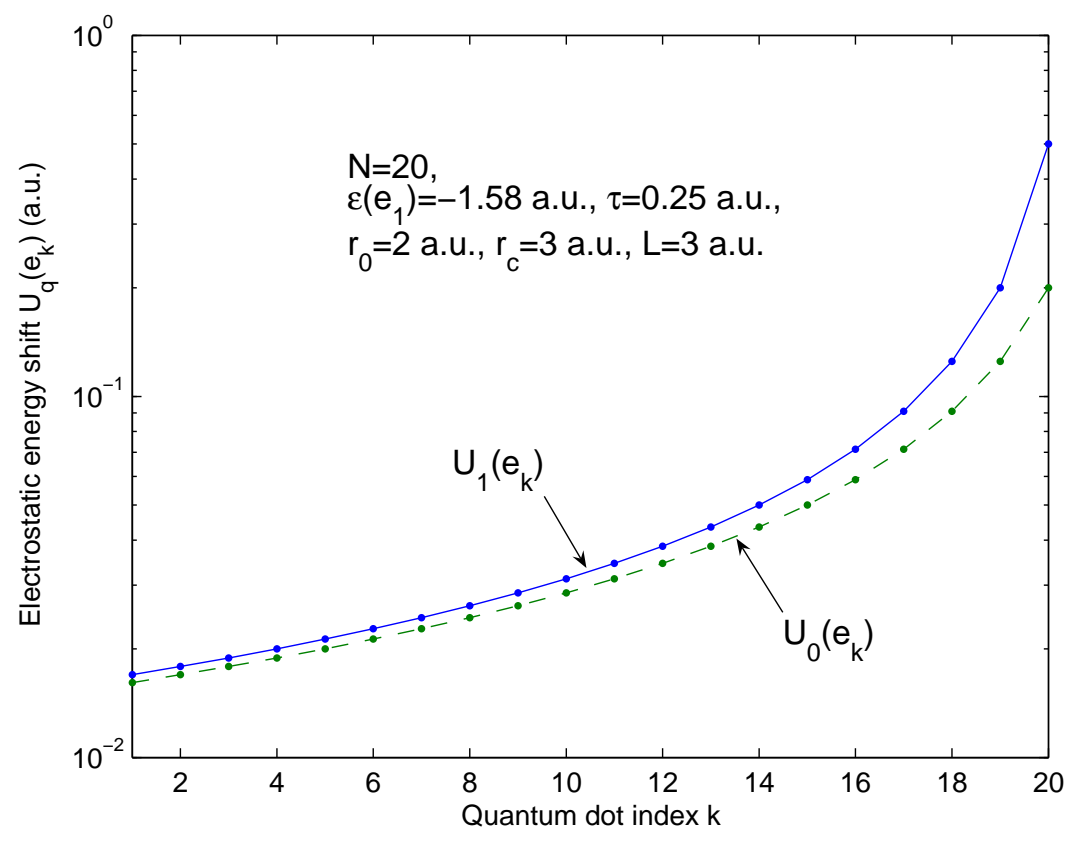

Figure 2 
Charge qubit entanglement in an array of quantum dots

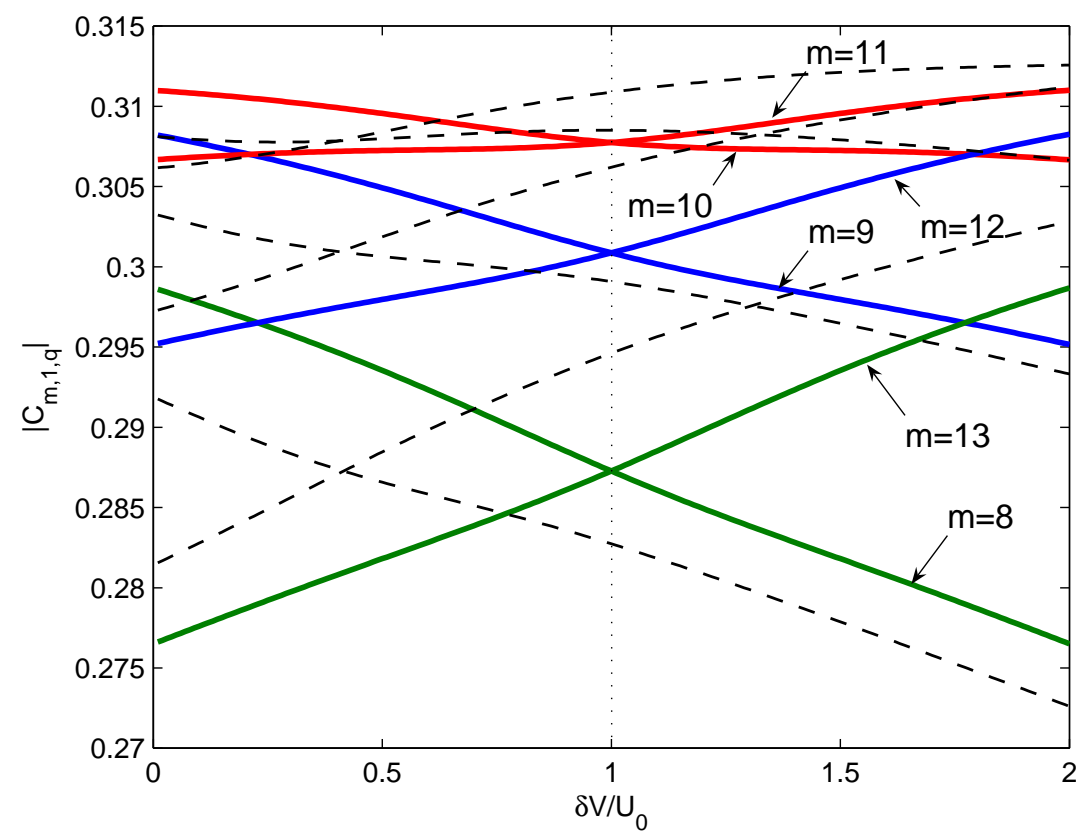

Figure 3 (a)

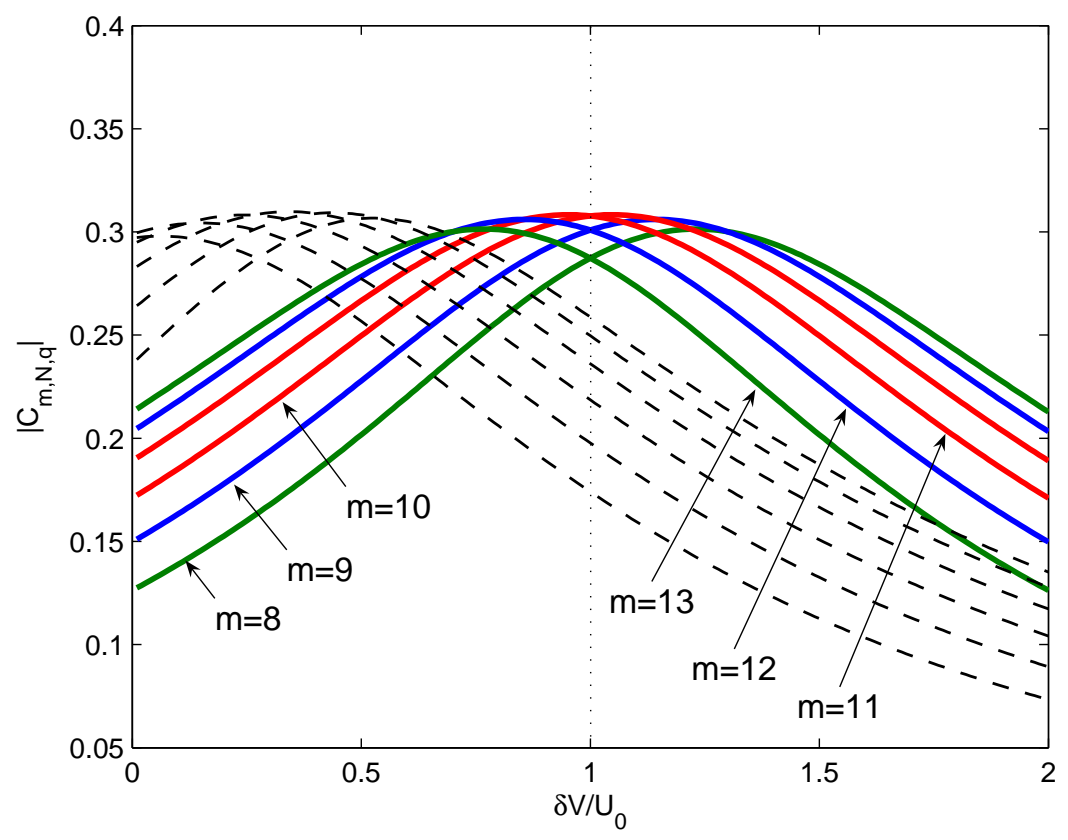

Figure $3(\mathrm{~b})$ 


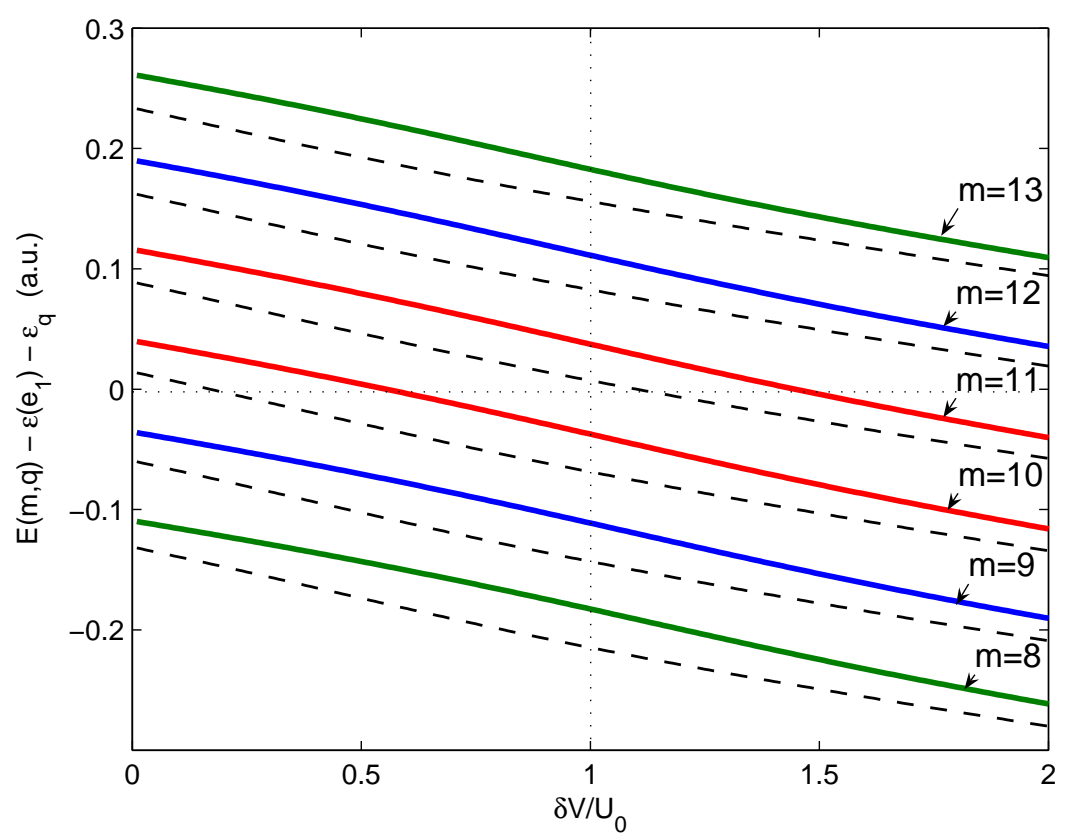

Figure 3 (c)

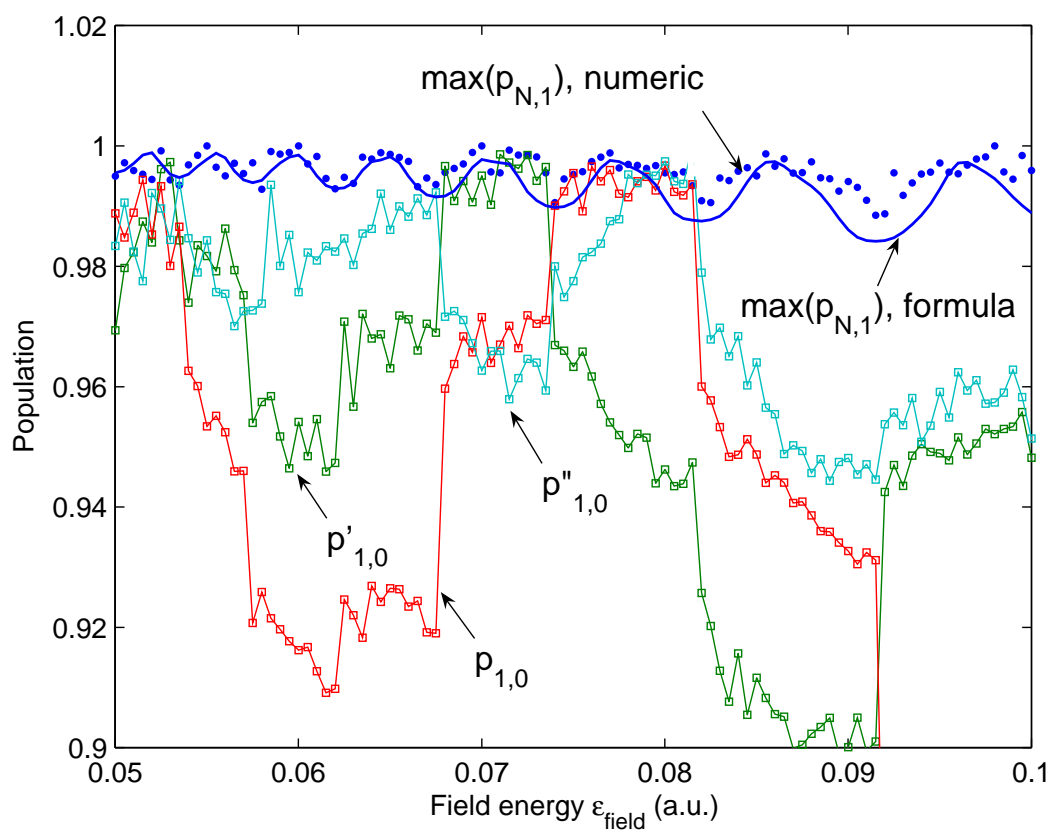

Figure 4 
Charge qubit entanglement in an array of quantum dots

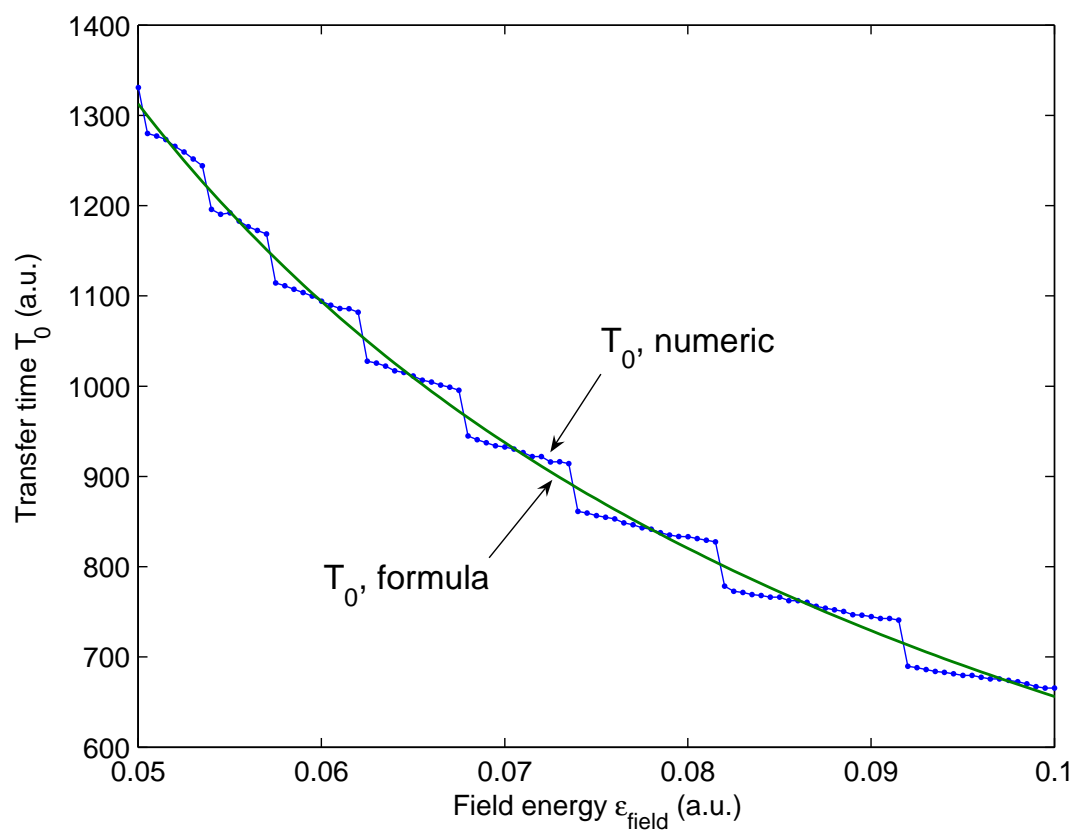

Figure 5

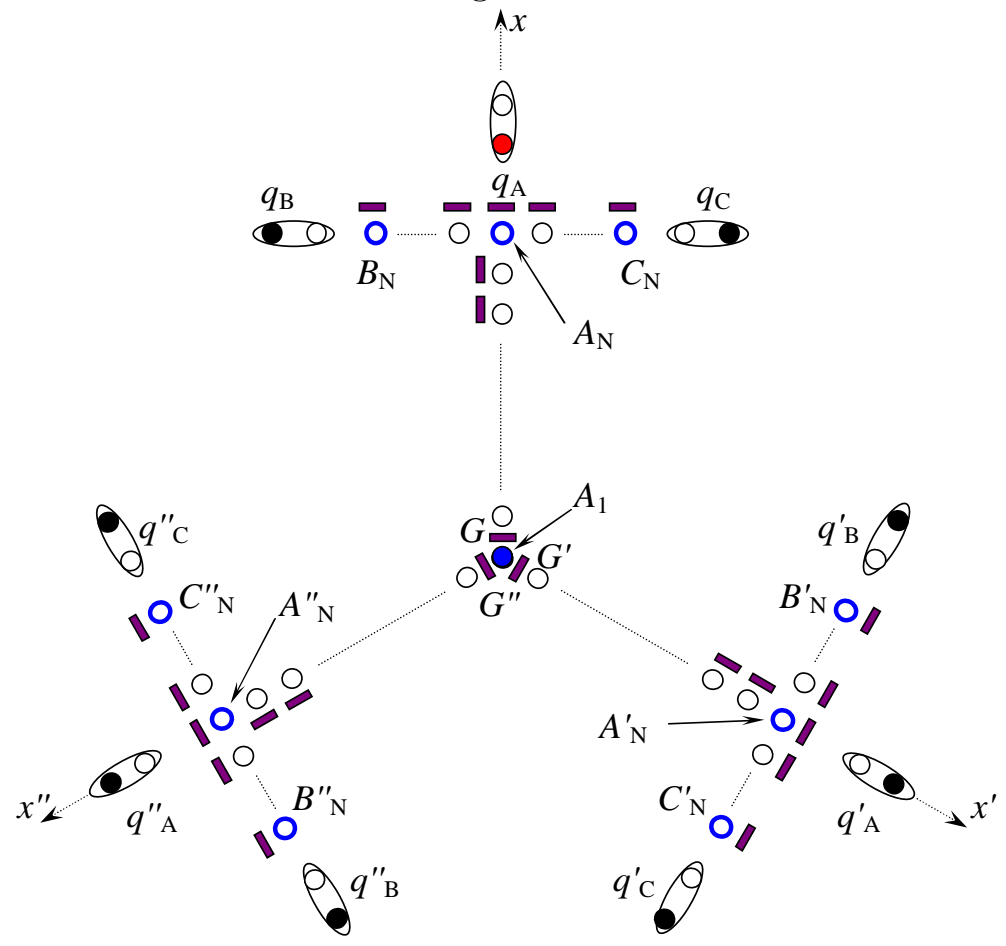

Figure 6 


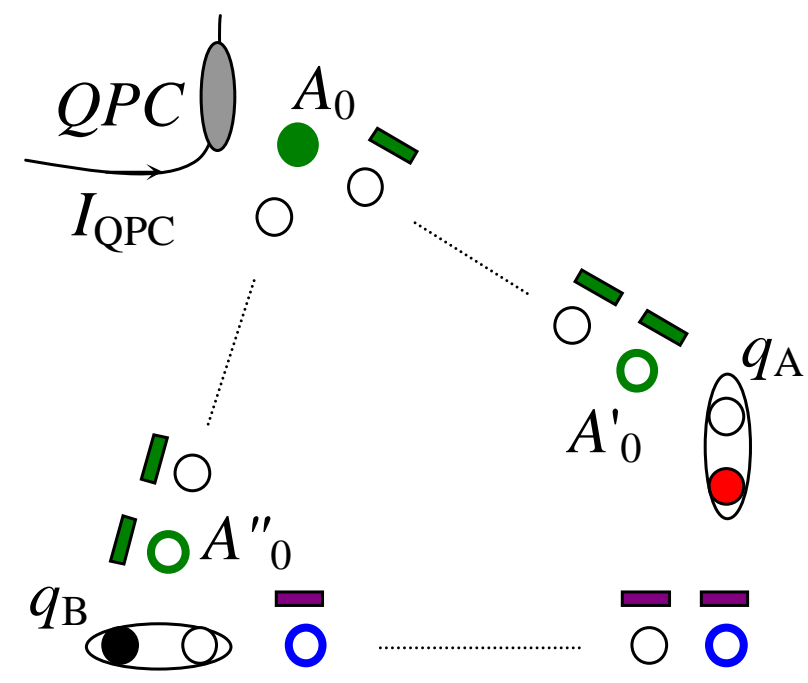

Figure 7 